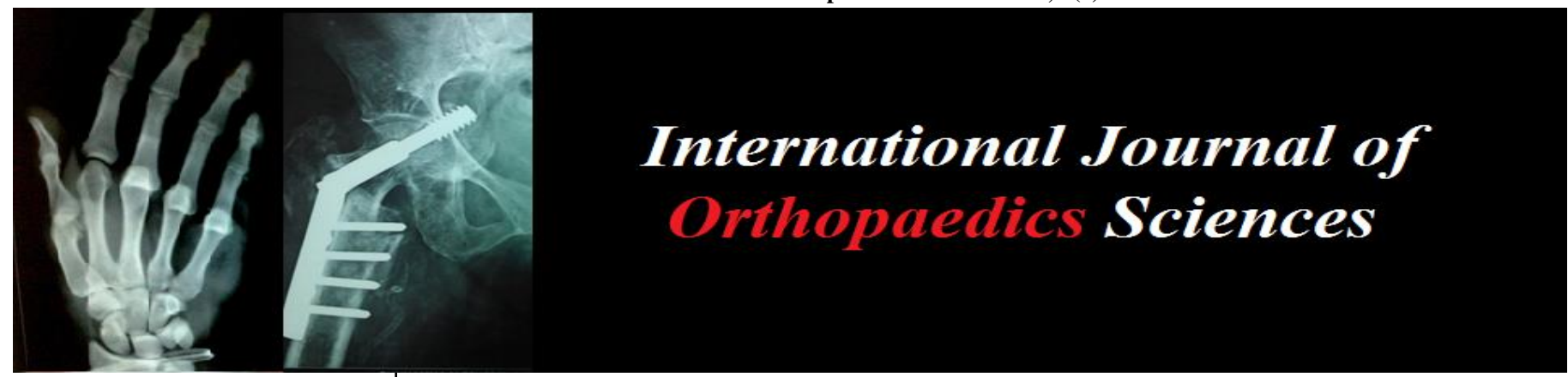

E-ISSN: 2395-1958

P-ISSN: 2706-6630

IJOS 2019; 5(4): 1070-1075

(C) 2019 IJOS

www.orthopaper.com

Received: 07-08-2019

Accepted: 09-09-2019

Dr. Surjit Kumar Kaparthy Junior Resident, Dept. of Orthopedics, Mamata Medical College, Khammam, Telangana, India

Dr. Balakrishnakanth P Associate Professor, Dept. oF Orthopedics, Mamata Medical College, Khammam, Telangana, India
Corresponding Author: Dr. Balakrishna Sir Associate Professor, Dept. of Orthopedics, Mamata Medical College, Khammam, Telangana, India

\section{A comparative study of functional outcome of fractures of both bone forearm treated by locking compression plate and dynamic compression plate}

\author{
Dr. Srujit Kumar Kaparthy and Dr. Balakrishnakanth P
}

DOI: https://doi.org/10.22271/ortho.2019.v5.i4r.1835

\section{Abstract}

Background: The forearm, being a component of upper limb serves important movements that are important in activities of daily living. The forearm, in combination with the proximal and distal radioulnar joints, allows pronation and supination which in turn helps hand, to perform multi axial movements ${ }^{[1]}$. With mechanized farming in India and industrialization, fractures of forearm bones have become more common. Fracture of the forearm bones may result in severe loss of function unless adequately treated. Hence good anatomical reduction and internal fixation of these fractures is necessary to restore function ${ }^{[2]}$. Closed reduction which was employed in earlier days yielded unsatisfactory results from either non union or loss of motion. Despite the combination of these different treatment techniques no compromises were made with regard to application as a compression plate or as a bridging device in the form of an internal fixation. LCP (Locking compression plate) is a product of these combinations and is in line with the latest plating techniques, the aim of which is to achieve the smallest surgical incision and to preserve blood supply to the bone and adjacent soft tissues and stability at the fracture site ${ }^{[6]}$. LCP has got features of both LC-DCP and a PC-Fix as it uses screw heads that are conically threaded on the undersurface and create an angular stable plate screw device 6. This type of plate fixation relies on the threaded plate-screw interface to lock the bone fragments in position and do not require friction between the plate and bone as in conventional plating ${ }^{[6]}$. The functional outcome was certified using "Anderson et $a l$., scoring system", the variables taken into consideration were

a. Union of the fracture,

b. Range of elbow and wrist movements ${ }^{[7]}$.

c. Extent of functional capacity reached

To conclude, satisfactory reduction of displaced fractures of the forearm bones is difficult to achieve by closed methods and if achieved, it is hard to maintain. So with open reduction and internal fixation using compression plate, it is possible to achieve perfect fracture reduction, rigid fixation, better bone healing and early mobilization.

Keywords: Radius, ulna, locking compression plate, dynamic compression plate

\section{Objectives}

- To compare and study functional outcome of forearm bone fractures after open reduction and internal fixation with DCP and LCP.

- To compare and study fracture union rate

- To compare and study complications of surgery

\section{Methods}

In this study 30 patients with forearm fractures, were treated by open reduction and internal fixation, of which 15 patients with $3.5 \mathrm{~mm}$ dynamic compression plate (DCP) and screws and 15 patients with $3.5 \mathrm{~mm}$ locking compression plate (LCP) and locking screws, in patients with displaced fractures of the shaft of forearm bones. This study was conducted from October 2017 to September 2019 


\section{Inclusion Criteria}

- Adults, both males and females

- Patients above the age of 18 years

- Patients fit for surgery and willing to participate in the study

- Radiologically diagnosed displaced diaphyseal fractures of both bone of forearm (AO classification-type A3, B3 and type $\mathrm{C}$ )

\section{Exclusion criteria}

- Fracture of forearm bones in children

- Patient not willing for surgery

- Patient unfit for surgery

- Isolated diaphyseal fracture of ulna or radius

- Patients associated with ipsilateral limb injury

- Patients lost in follow up period

- Diaphyseal fractures associated with compartment syndrome or requiring neurovascular repair

\section{Operative Techniques}

\section{Dynamic Compression Plate}

\section{Operative procedure}

Usually radius was fixed first, however the bone which was less comminuted and more stable was fixed first and later the other bone was fixed after identifying the fracture ends, periosteum was elevated and fracture ends were cleaned. Fracture was reduced. The contoured plate is applied to the bone with middle portion placed over the fracture, and held with reduction forceps for short oblique, of transverse fracture. A plate hole is left vacant for angled lag screw through the plate in case of oblique fractures. This hole is used for interfragmentary compression of a lag screw. A plate of at least 6 holes was chosen and longer plates were used in spiral, segmental and comminuted fractures for upper third radial fractures, the plate was fixed dorsally. For middle third, the plate was fixed dorsolateral and for distal radial fractures the plate was fixed on the volar aspect. In ulnar fractures, plate was applied over the posterior surface of Ulna4. The arrow of the neutral drill guide points towards the fracture. $2.5 \mathrm{~mm}$ drill bit is used for drilling a hole through both cortices and with depth gauge, appropriate $3.5 \mathrm{~mm}$ screw length is determined. The plate is pulled towards the fracture to place first eccentric screw. The second screw hole for axial compression is drilled in the fragment which forms an acute angle near the plate. In the direction of fracture line to be compressed. a lag screw will be inserted. Tightening of the two screws produces axial compression. The lag screw is applied by subsequently over drilling $(3.5 \mathrm{~mm})$ the near cortex to create a gliding hole. If compression is sufficient the remaining screws are applied one by one, alternating from one side to the other. In case of porotic, comminuted and/or small bones, long screws and/or a longer plate were used. Finally tightening of all the screws. hemostasis is maintained, the wound is closed in layers over a suction drain and sterile dressing is applied.
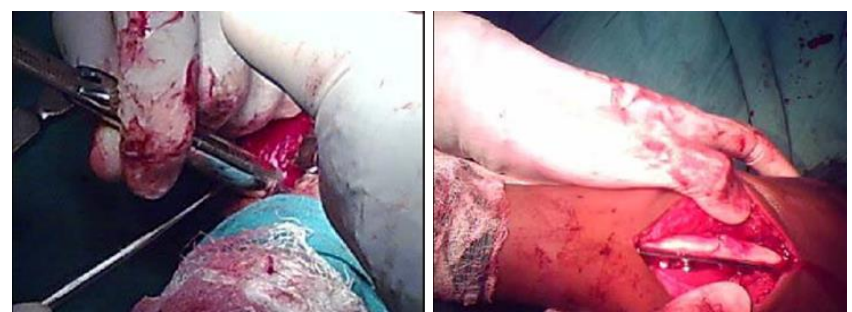

Fig 1: fracture site

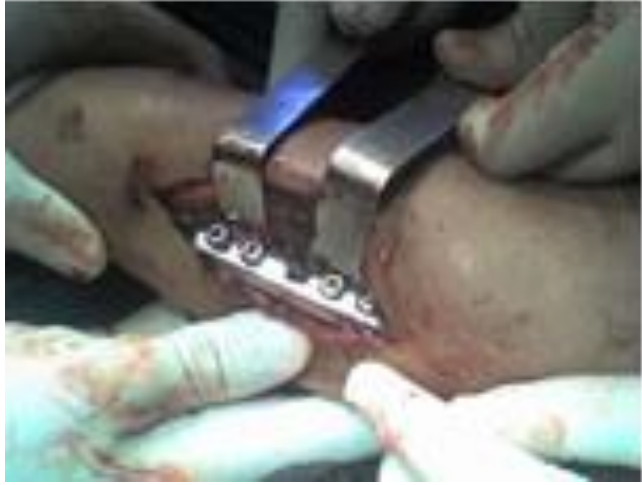

Fig 2: DCP in position
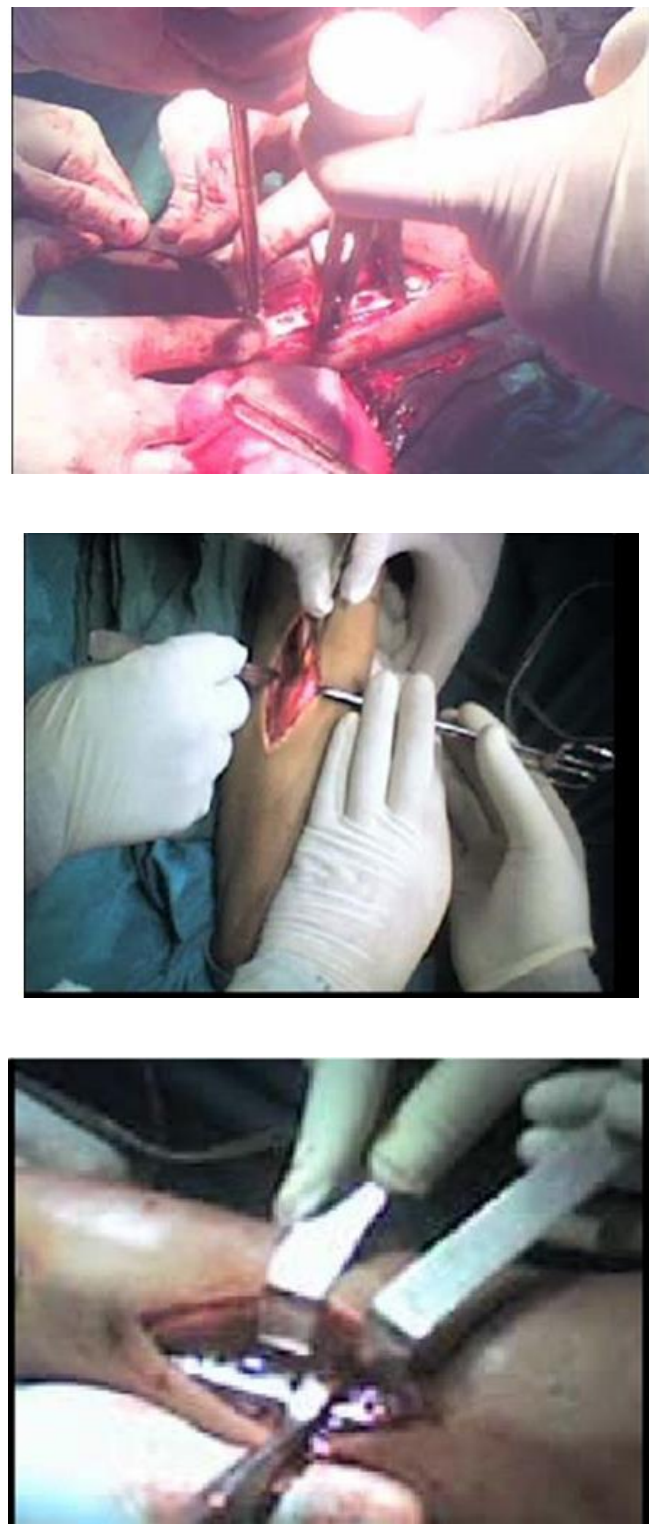

Fig 5: Intra Operative Images of Dcp Plating

\section{Locking Compression Plate}

After identifying the fracture ends, periosteum was not elevated and fracture ends were cleaned. With the help of reduction clamps fracture was reduced and held in position. The plate was then applied A plate of at least 6 holes was chosen and longer plates were used in spiral, segmental and comminuted fractures. For upper third radial fractures, the plate was fixed dorsally. For middle third, the plate was fixed dorsolateral and for distal radial fractures the plate was fixed 
on the volar aspect. In ulnar fractures, plate was applied over the posterior surface of ulna. A drill sleeve for locking screw is fixed in the hole, near the fracture site, and $2.7 \mathrm{~mm}$ drill bit is use to drill both the cortex of the bone, the sleeve is removed and the screw length is measured with depth gauge. A $3.5 \mathrm{~mm}$ locking screws are then inserted, as the locking screws are of self tapping, tapping of the screw hole is not done. After adaptation of the fragments, a screw hole for axial compression is drilled in the fragment which forms an acute angle near the plate. Here the load guide is used with the arrow pointing towards the fracture line to be compressed. At this position, a lag screw will be inserted for axial compression. The lag screw is applied by subsequently over drilling $(3.5 \mathrm{~mm})$ the near cortex to create a gliding hole. The lag screw and remaining screws are inserted. Once stable fixation is achieved and hemostasis secured meticulously, the wound is closed in layers over a suction drain and sterile dressing is applied

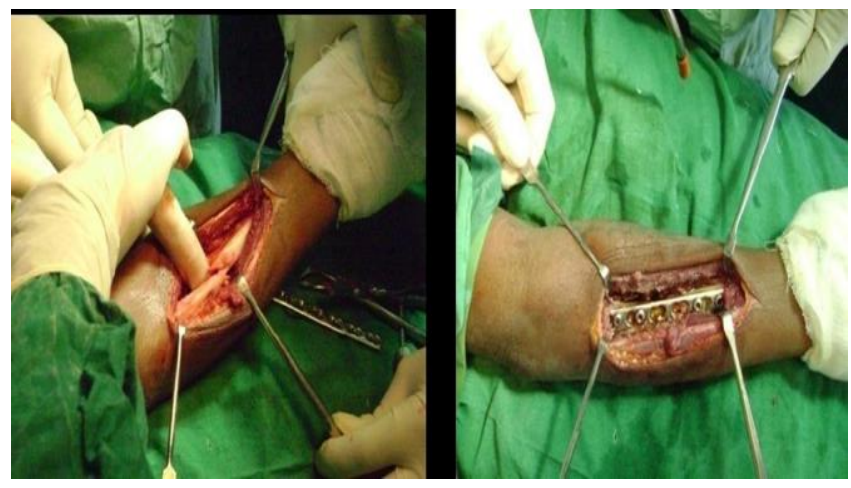

Fig 6: fracture site

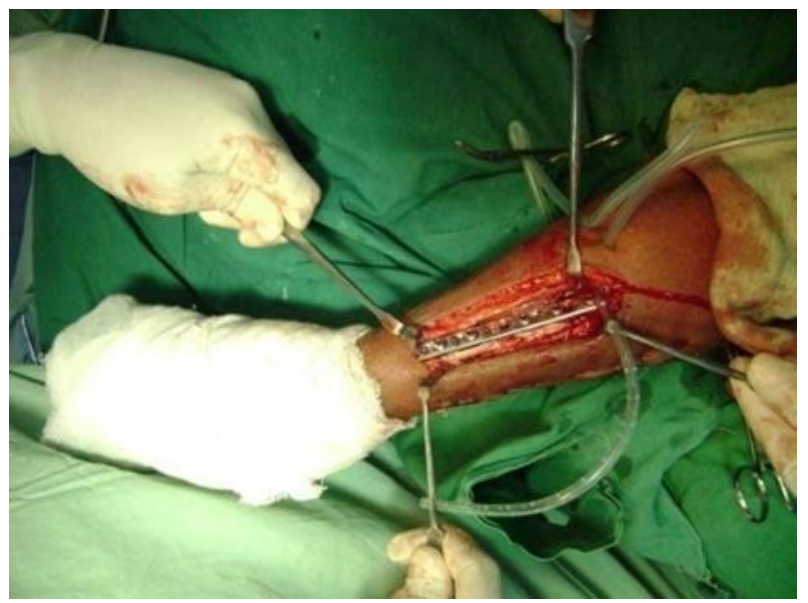

Fig 7: Intra Operative Pics of Lcp Plating

\section{Post-operative treatment}

Crepe bandage was applied over the affected forearm and either pre op posterior slab was continued or arm pouch was given depending upon the requirement. Limb is elevated and active movement of the fingers and elbow joint is encouraged. Suction drain was removed after 48 hours and Wound was inspected. Check X ray AP and Lateral view was taken at that time. Antibiotics and analgesics were continued till the time of suture removal which was done on 10-12 postoperative day. On discharge patient was advised physiotherapy of shoulder, elbow, wrist and finger movements. They were told not to lift heavy weight or exert the affected forearm.

\section{Follow-up}

The patients were followed regularly at monthly interval for first three months then every three months depending upon the outcome. The patients were evaluated based on "Anderson et al. 1" scoring system. Elbow movements and wrist movements were noted and the union was assessed radiologically. The fracture is said to be united when there was presence of periosteal callus bridging the fracture site and trabeculation extending across the fracture line Anderson et al. had a follow up from 4 month to 9 years with an average of 3 years7. Moed in his series followed patients from 12 months to 9 years with an average of 3 years.35 Chapman series had follow up which ranged from 6 months to 48 months with average of 12 months.39 Present study had a follow up which ranged from 5 months to 18 months with an average mean of 12 months, which is comparable to Chapman series but other series had longer follow up.

\section{Results}

Table 1: analysis

\begin{tabular}{|c|c|c|c|c|c|}
\hline \multicolumn{2}{|c|}{ Implant used } & $<13$ & 13-24 & Union in weeks >24 & Non-union \\
\hline DCP & Frequency & & 12 & 1 & 0 \\
\hline Percent & 80 & 6.66 & & 13.33 & 0 \\
\hline LCP & Frequency & & 13 & 1 & 0 \\
\hline Percent & 86.66 & 6.66 & & 6.66 & 0 \\
\hline Total & Frequency & & 25 & 3 & 0 \\
\hline Percent & 83.33 & 6.67 & & 10 & 0 \\
\hline
\end{tabular}

Chi-square $=0.373$

A non-significant association was observed between implant used and weeks for union (d.f=2; $P<0.83$ ). From the table, it is clear that most of the implants had the weeks for union $<13$, few cases had the weeks for union13-17, >24 weeks for union, two cases were reported from DCP and one for LCP. Between weeks for union and implant used, a non-significant association was observed as Chi-square value (0.373) revealed a non-significant value (d.f=2; $P<0.83)$, indicating that weeks for union for cases in different implants used was statistically same. From the table it is clear that 25 patients, 12 fixed with DCP and 13 with LCP had union in less than 13 wks and Two cases in DCP and one case in LCP had union > 24 weeks.Also indicating that patients treated with LCP had faster union compared to those treated with DCP.

Table 2: Anderson et al. scoring system

\begin{tabular}{|c|c|c|c|}
\hline Results & Union & Flexion/Extension at & Supination and \\
\hline $\begin{array}{c}\text { elbow joint and } \\
\text { wrist Excellent }\end{array}$ & Present & $\begin{array}{c}\text { pronation of forearm } \\
<100 \text { loss }\end{array}$ & $<25 \%$ loss \\
\hline $\begin{array}{c}\text { Satisfactory } \\
\text { Unsatisfactory }\end{array}$ & $\begin{array}{c}\text { Present } \\
\text { Present }\end{array}$ & $<200$ loss & $<50 \%$ loss \\
\hline
\end{tabular}

Failure Non union with or without loss of motion

\section{Discussion}

Diaphyseal fractures of forearm are seen most commonly in middle aged subjects. Males show high incidence of fractures as they are often engaged in agriculture, industrial work and motor vehicle driving. The cause of fractures is mostly due road traffic accident and fall on an outstretched hand. Majority of the fractures were transverse/short oblique in the middle third region of forearm. With the use of AO/ASIF 3.5 $\mathrm{mm}$ DCP and $3.5 \mathrm{~mm}$ LCP for acute diaphyseal fractures of forearm, rigid and anatomical fixation can be achieved. With use of DCP and LCP, distraction forces leading to separation fracture fragments like seen in interlocking nail for upper limb is not possible. Radial bowing is very important for normal supination and pronation. This can maintained very 
well with compression plates. A minimum of 6 cortices should engage in each fracture fragment. It is better to use longer plates like a bridge plate in case of comminuted oblique fractures. Radius and Ulna are approached separately to avoid extensive soft tissue dissection and resulting complication. Post operatively with DCP and LCP fixation additional supportive measures may not be required after soft tissue healing and shoulder elbow and wrist movements can be started early. This helps prevent muscle atrophy and joint stiffness. It is very much possible in intelligent and cooperative patients. However all patients should be curtailed from lifting heavy weights till union of fracture. Almost all fractures in our study united by 4-6 months.

\section{Complications}

\section{Infection}

Infection after operative treatment of forearm fractures is not uncommon. Acute infections require standard treatment with irrigation and debridement, and the hardware should not be removed if the fixation is stable. When the hardware is stable, it maintains length, rotation, and alignment and assists in wound care. In late infections, treatment is similar, and plate removal may be performed if the fracture is healed.

\section{Nerve Injury}

Nerve injuries associated with fractures of both bones of the forearm are uncommon in closed fractures. If the nerve injury occurs as the result of treatment, recommendations for agement are as follows. Incomplete iatrogenic nerve injuries can be observed for several weeks or months to determine if recovery will occur. If there is no evidence of recovery by three months, exploration is indicated. Complete iatrogenic nerve injuries should be explored early (within a few days) if the nerve was not observed at the time of the primary operation and neurologic function does not rapidly, progressively and completely recover. Early exploration is desirable in such cases to be certain that the nerve was not damaged by plating or suture placement. If the nerve had in fact been exposed and visualized throughout the operation (including just before closure) and the. Surgeon is confident that the nerve was not damaged, then observation for three months as described above with the expectation of recovery is the appropriate management. Anterior interosseous nerve palsy caused by a constrictive dressing, a complication that is preventable in most cases by the application of a postoperative dressing that provides support for the limb while at the same time allows for the soft tissues to swell. Posterior interosseous nerve is at danger during dorsal (Thompson) approach to proximal radius. In our series we had two cases $(6.66 \%)$ of weakness of extensor muscles of forearm immediately following surgery. Both the cases had neuropraxia of posterior interosseous nerve during following dorsal Thompson approach.

\section{Vascular Injury}

If the collateral circulation of the forearm is good, and if either the radial or the ulnar artery is functioning, viability of the hand and forearm is usually not in jeopardy. It is rare to have both vessels lacerated except in open fractures in which a traumatic near-amputation has occurred 55, 56, 95.

\section{Compartment syndrome}

This can occur either after trauma or after surgery on the forearm bones. They are usually due to faulty hemostasis or closure of the deep fascia57. They can usually be avoided by releasing the tourniquet before wound closure to make sure hemostasis is adequate, by closing only the subcutaneous tissue and skin, and by using suction drains.

In our series we had no case to develop compartment syndrome possibly because we thoroughly checked for adequate hemostasis and use of suction drains and closure of the wound only through subcutaneous tissue and skin.

\section{Radioulnar Synostosis}

The most common cause of posttraumatic radial-ulnar synostosis is an operatively treated forearm fracture. Patients with high-energy, comminuted, open fractures appear more likely to develop this complication. Monteggia and proximal forearm fractures also appear to have a higher incidence of this complication. The use of screws protruding through the opposite cortex and use of bone graft also increase the incidence of synostosis. Additionally, radial-ulnar synostosis is described as a consequence of soft tissue injury, reconstructive procedures, any trauma causing hematoma formation between the radius and ulna, or injury to the interosseous membrane. Patients with closed head injuries (skull/cranial trauma) appear to be more prone to this complication, presumably for the same reason that they develop heterotopic ossification.

\section{Radioulnar Synostosis}

The most common cause of posttraumatic radial-ulnar synostosis is an operatively treated forearm fracture. Patients with high-energy, comminuted, open fractures appear more likely to develop this complication. Monteggia and proximal forearm fractures also appear to have a higher incidence of this complication. The use of screws protruding through the opposite cortex and use of bone graft also increase the incidence of synostosis. Additionally, radial-ulnar synostosis is described as a consequence of soft tissue injury, reconstructive procedures, any trauma causing hematoma formation between the radius and ulna, or injury to the interosseous membrane. Patients with closed head injuries (skull/cranial trauma) appear to be more prone to this complication, presumably for the same reason that they develop heterotopic ossification.

\section{Malunion}

Malunion was quantified by measurement of the amount and location of the maximum radial bow in relation to the contralateral, normal forearm. The effect of residual angulations from simulated fractures of both bones of the forearm on the potential, for range of rotation of the forearm 97 and for limitations of pronation and supination specifically little significant loss of forearm rotation resulted from angulations of 10 degrees in any direction. With 20 degrees of angulation, results in statistically significant and functionally important loss of forearm rotation.

\section{Non-union}

Failure to show any radiological evidence of union even after 9 months of surgery and required additional procedure of bone marrow injection or bone grafting or revision fixation were deamed as nonunion. They appear to have been caused by infection or errors in technique. Accurate open reduction and rigid internal fixation will prevent these complications.

In our series we had $100 \%$ union rate that is zero cases $(0 \%)$ had nonunion. In our series out of 15 cases treated with DCP 12 cases had union achieved by 13 weeks, one case union occurred by 20 weeks two case had union by 28 weeks which 
were deamed as delayed union but none $(0 \%)$ had non union While out of 15 cases treated with LCP 13 cases had union by 13 weeks while one case had union by 19 weeks and one case had union by 27 weeks while none $(0 \%)$ cases had nonunion.

\section{Refracture}

Two other complications that have been seen with compression plates are refractor (if the plate is removed too early) and fracture at the end of the plate from additional trauma. Plates provide very rigid fixation, and the normal stresses acting over the bone beneath the plate are reduced. If the plate is removed early, minor trauma can cause a refracture at or near the site of the original fracture. Refracture should be included among the risks of plate removal. Ideally, plates should be left in place at least 18 months because refracture is more common if removal is performed sooner than this. Patients should be informed that refracture can occur for several months after plate removal and they should avoid impact-type activities for 8 weeks after surgery in our series we had no cases of implant removal done until the time of analysis of the study.

\section{Soft tissue contracture}

Contracture of the interosseous membrane, proximal radioulnar joint or distal radioulnar joint either in isolation or combination will result in significant loss of forearm rotation. Prevention of contracture is achieved by rigid fixation and early motion. 99 In our series no patient developed any soft tissue contracture. This could be because In our series all the patients were mobilized once the surgical site was healed.

In comparison between the two groups treated with DCP and LCP we found that there is no significant difference in possibility of complications or outcome of those complications.

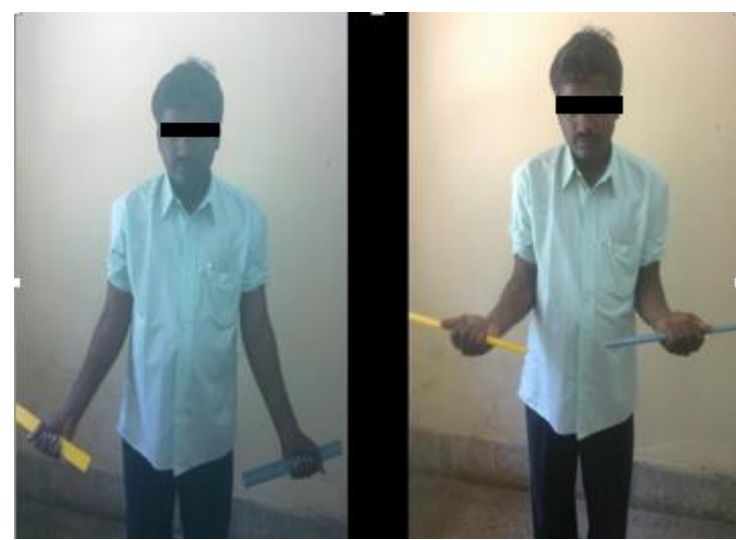

Fig 8: clinical pictures in post op

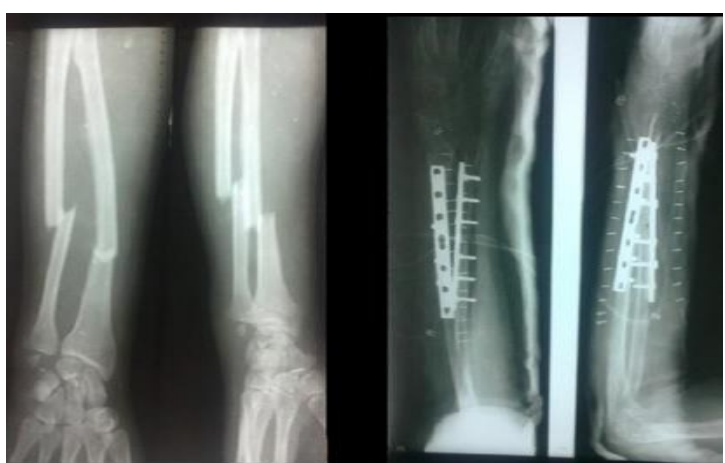

Fig 9: Case Showing Pre Op, Post Op at 1 Month and 3 Months

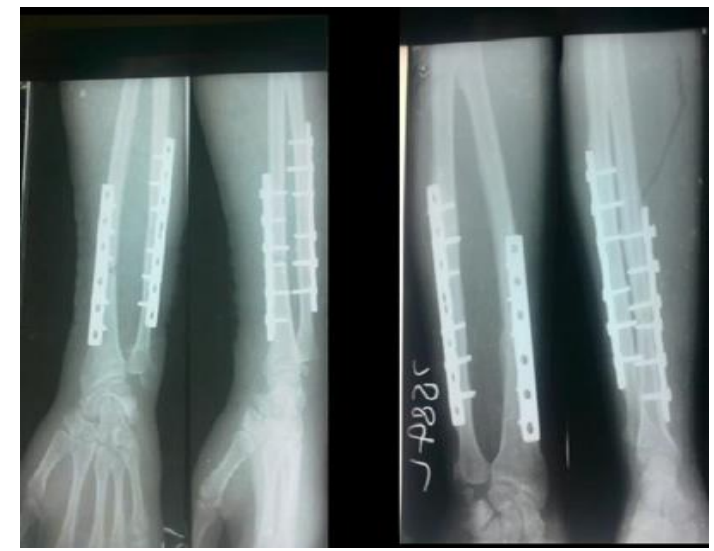

Fig 10: Melorheostosis and Osteopoikilosis

\section{Interpretation and Conclusion}

The range of motion was determined and Anderson et al., scoring system was used as a measure for the functional outcome ${ }^{[7]}$. Chapman et al. reported $36(86 \%)$ cases as excellent, $3(7 \%)$ satisfactory, 1(2\%) unsatisfactory and 2(5\%) failure 39. Anderson et al. reported about 54(50.9\%) cases as excellent, $37(34.9 \%)$ satisfactory, 12(11.3\%) unsatisfactory and $2(2.9 \%)$ failure ${ }^{[7]}$. Frankie et al. reported $98 \%$ cases as excellent while $2 \%$ as satisfactory ${ }^{[66]}$. In our series we had 25 $(83.33 \%)$ cases with excellent results, $5(16.67 \%)$ satisfactory and $0(0 \%)$ cases of unsatisfactory result and $0(0 \%)$ cases of failure. In comparison between the two groups treated with DCP and LCP we found that there is no significant difference in functional outcome.

\section{References}

1. Morgan William J, Thomas P. Breen: Complex fractures of forearm. Hand clin. 1994; 10(3):375-390.

2. Richards RR. Fractures of the shafts of the radius and ulna. In. Bucholz RW, Heckman JD, editors. Rockwood and Green's fractures in adults. 5th ed. Philadelphia, USA: Lippoincott Williams and Wilkins, 2001, 869-917.

3. Dodge, Herbert S, Gerald W. Cady, Treatment of fractures of the radius and ulna with compression plates. J Bone Joint Surg. 1972: 54-A(6):1167-1176.

4. Bagby GW, Bone J, Joint Surg Am. 1977; 59:625631.Compression bone-plating: historical considerations

5. Texhammer R. AO/ASIF Instrumentation. Chapter-6 in AO/ASIF Instruments and implantsedn, Texhammer $\mathrm{R}$ and C. Colton, Berlin, Springer Verlag. 1994; 2:84-86.

6. Leung F, Chow SP: Locking compression plate in the treatment of forearm fractures A prospective study. J Orthop Surg. (Hong kong). 2006; 14(3):291-4.

7. Anderson LD, Sisk D, Tooms RE, Park WI. Compression plate fixation in acute diaphyseal fractures of the radius and ulna J. Bone Joint Surg. Am., Apr. 1975; 57:287.

8. William a Clark. History of fracture treatment. J.bone Joint Surg. Am.1937; 19:47-63.

9. Colton C. History of Osteosynthesis. Chapter-2, in AO/ASIF Instruments and implants 2nd edn, Texhammer R and C. Colton, Berlin, Springer Verlag, 1994, 3.

10. Patrick J. A study of supination and pronation with special reference to the treatment of forearm fractures. J Bone Joint Surg. 1946; 28:737-748.

11. Chandler RN. Principles of Internal Fixation. Chapter-3, in Fractures in Adults, Vol. Edn., Rockwood C. A. Jr. et al., Philadelphia; Lippincott Raven. 1996: 1(4):159.

12. Evans EM, Rotational deformity in the treatment of fracture of both bone forearm, J Bone \& Joint Surg. 
1945; 24:373-379.

13. Smith H, Sage FP. Medullary fixation of forearm fractures. J. Bone Joint Surg. Am., Jan. 1957; 39:91-188.

14. Crenshaw, Andrew H. Fractures of shoulder girdle, Arm and Forearm. Chapter-, in Campbells Operative Orthopedics, Edt. Canaled, S. Terry, Mosby. 2003; 49:3042-3058.

15. Perren SM. The concept of biological plating using the limited contact dynamic compression plate $\{$ LC-DCP $\}$, Injury. 1991; 22(1):1-41.

16. Smith JEM. Internal Fixation in the Treatment of Fractures of the shaft of Radius and Ulna in Adults, $\mathrm{J}$ Bone \& Joint Surg. 1959; 41(1):122-131.

17. Burwell HN, Charnley DA. Treatment of Forearm fractures in adults with particular reference to plate fixation, J. Bone \& Joint Surg. 1964; 46(3):404-424.

18. Sarmiento Augusto, Cooper SJ, Sinclair FW. Forearm fractures. J Bone \& Joint Surg. 1975; 57-A(3):297-304.

19. Watson-Jones. Fracture and Joint injuries. 6th ed. Churchill Livingstone, 1982.

20. Schemitsch, Emil H, Richards RR. The effect of malunion on functional outcome after plate fixation of fracture of both bones of forearm in adults. $J$ Bone \& Joint surg. 1992; 74-A(7):1068-1078. 